\title{
Epifisiólise em paciente de 22 anos de idade com hipogonadismo hipogonadotrópico congênito: Relato de caso*
}

\section{Epiphysiolysis in a 22-Year-Old Patient with Congenital Hypogonadotropic Hypogonadism: Case Report}

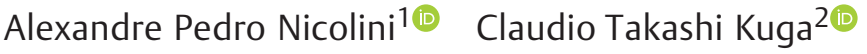 \\ ${ }^{1}$ Centro de Traumatologia do Esporte, Escola Paulista de Medicina, \\ Universidade Federal de São Paulo, São Paulo, SP, Brasil \\ 2 Departamento de Ortopedia e Traumatologia, Hospital Geral de \\ Pedreira, São Paulo, SP, Brasil \\ Endereço para correspondência Alexandre Pedro Nicolini, M.D., \\ Centro de Traumatologia do Esporte - Universidade Federal de São \\ Paulo/Escola Paulista de Medicina, Rua Estado de Israel, 636, \\ São Paulo, SP, Brasil (e-mail: apnicolini@uol.com.br).
}

Rev Bras Ortop

\section{Resumo \\ Palavras-chave \\ - epífise deslocada \\ - síndrome de Kallmann \\ - cabeça do fêmur \\ - epifisiólise}

A epifisiólise é uma doença relativamente comum na população adolescente (de 9-16 anos), entretanto rara na população adulta. Se caracteriza pelo escorregamento metáfiso-epifisário do fêmur proximal não-traumático. Quando ocorre nessa população, está associada a alguma doença que retarda o desenvolvimento sexual e fechamento fisário, como doenças endocrinológicas ou tumores cerebrais. O objetivo do presente estudo é relatar um caso de epifisiólise numa paciente com 22 anos de idade e hipogonadismo hipogonadotrófico. Existem apenas 63 casos relatados na literatura mundial sobre epifisiólise na população adulta.

Epiphysiolysis is a relatively common disease in the adolescent population (9-16 years); however, it is rare in the adult population. It is characterized by non-traumatic proximal femur slipping. When it occurs in this population, it is associated with some disease that slows sexual development and physis closure, such as endocrine diseases or brain tumors. The aim of the present study is to report a case of epiphysiolysis in a 22-year-old patient with hypogonadotropic hypogonadism. There are only 63 cases reported in the world literature on epiphysiolysis in the adult population.
Trabalho desenvolvido no Centro de Traumatologia do Esporte Universidade Federal de São Paulo, Escola Paulista de Medicina, São Paulo, SP, Brasil. recebido

30 de Agosto de 2020

aceito

01 de Dezembro de 2020
DOI https://doi.org/

$10.1055 / \mathrm{s}-0041-1726071$. ISSN 0102-3616. (c) 2021. Sociedade Brasileira de Ortopedia e Traumatologia. All rights reserved.

This is an open access article published by Thieme under the terms of the Creative Commons Attribution-NonDerivative-NonCommercial-License, permitting copying and reproduction so long as the original work is given appropriate credit. Contents may not be used for commercial purposes, or adapted, remixed, transformed or built upon. (https://creativecommons.org/ licenses/by-nc-nd/4.0/)

Thieme Revinter Publicações Ltda., Rua do Matoso 170, Rio de Janeiro, RJ, CEP 20270-135, Brazil 


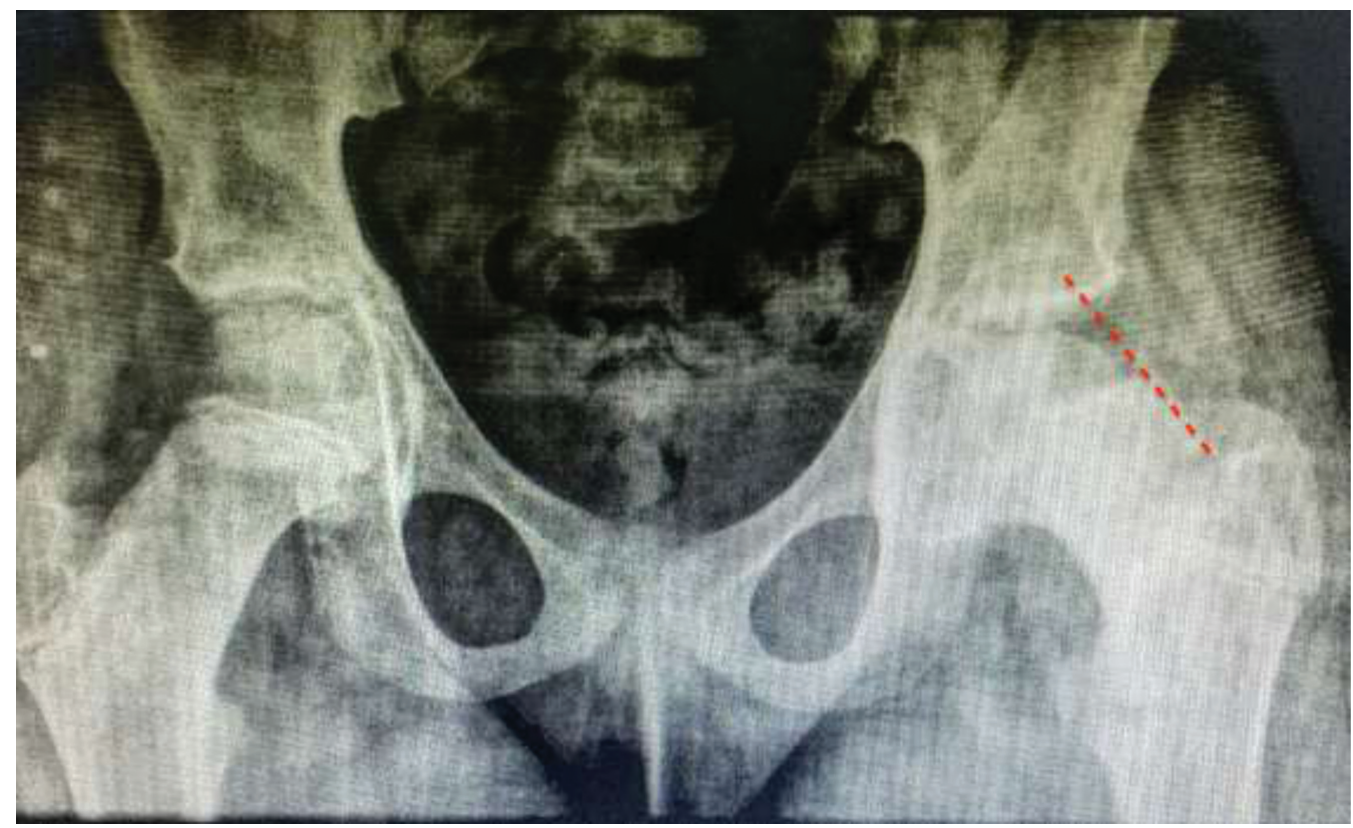

Fig. 1 Radiografia frontal demonstrando escorregamento do lado E e sinal de Trethowan.

\section{Introdução}

Epifisiólise é uma das principais doenças que acometem o quadril do adolescente e se caracteriza pelo escorregamento metafisário femoral, não-traumático, ântero-lateralmente em relação à epífise, que continua centrada no acetábulo. ${ }^{1}$ Acomete principalmente pacientes na faixa etária entre $9 \mathrm{e}$ 16 anos (média de 12) $\cdot^{1-4}$ Ocorre por uma fise fragilizada (fatores histológicos, vasculares e endocrinológicos) submetida a uma sobrecarga mecânica., ${ }^{4,5}$

Apresenta incidência bastante variada na literatura, de 0,03/10.000 até 5/10.000 dependendo do trabalho, gênero, região e etnicidade. ${ }^{1-4,6,7}$ A epifisiólise acomete mais pacientes do sexo masculino $(2: 1,4)$ do que do sexo feminino e é mais incidente em negros e hispânicos. ${ }^{4,7}$

A ocorrência bilateral simultânea é de $7 \%{ }^{7}$ podendo chegar a $21 \%$ assincronicamente. Aproximadamente $90 \%$ dos escorregamentos contralaterais ocorrem dentro de 18 meses, ${ }^{4}$ e esta condição é mais incidente quando associada a doenças endocrinológicas. Nestes casos, a fixação contralateral é indicada, mesmo que o paciente esteja assintomático. ${ }^{2,4,5}$

Clinicamente, ocorre queixa de dor no quadril ou joelho e dificuldade de deambular, comumente insidiosa e raramente de maneira aguda. Frequentemente o diagnóstico é tardio ou negligenciado pelo quadro clínico frustro e incaracterístico, causando um tratamento tardio e pior prognóstico. ${ }^{4,8}$ Ao exame físico, há limitação da flexão e da rotação interna e o sinal de Drehmann (abdução e rotação externa durante a flexão passiva $)^{4}$ é característico.

Na radiografia (frente e Lauenstein), observa-se o escorregamento nos casos mais avançados e nos casos iniciais sinais indicativos, como alargamento e irregularidade da fise. São descritos dois métodos radiográficos para diagnóstico e mensuração do escorregamento: sinal de Trethowan - linha na superfície superior do colo femoral (linha de Klein) não cruza com a fise (-Figura 1), e mensuração do ângulo de Southwick (entre cabeça e diáfise no perfil) ( - Figura 2) ${ }^{4,8} \mathrm{~A}$ ressonância magnética é recomendada para casos iniciais ou dúvida diagnóstica.

A epifisiólise pode ser classificada de 3 maneiras: quanto à duração: aguda $<3$ semanas ou crônica $>3$ semanas; baseada nos sintomas (classificação de Loder): estável paciente consegue deambular, ou instável - paciente não consegue ambular mesmo com auxílio de muletas, ${ }^{2,4}$ e classificação radiográfica, pelo grau do escorregamento. ${ }^{2,4}$

De etiologia multifatorial, inúmeras doenças ou características possuem associação: obesidade, osteodistrofia renal, hipotireoidismo, hipogonadismo, hiperparatiroidismo, ${ }^{2-4}$ aumento da retroversão femoral, ${ }^{1,4}$ retroversão acetabular, ${ }^{4}$ aumento da obliquidade fisária $^{4}$ e privação sócioeconômica. ${ }^{9}$

O tratamento preconizado é a fixação in situ, provocando a epifisiodese. Quando ocorre redução do escorregamento há um aumento da ocorrência de necrose avascular da cabeça femoral (NACF) e condrólise, ${ }^{4,6}$ sendo estas as complicações mais comuns da doença. Após a fixação in situ, a taxa de NACF relatada na literatura é de $1,5 \%{ }^{4,6}$ Um total de $10 \%$ dos pacientes apresentará quadro de osteoartrose grave após os 20 anos de idade. ${ }^{4,6}$

A ocorrência é rara em adultos, com apenas alguns relatos na literatura. Quando acontece, sempre existe associação com alguma doença que atrasa a maturação sexual e, consequentemente, o fechamento fisário. ${ }^{10}$ As doenças mais comumente descritas associadas à epifisiólise em adultos são: hipopituitarismo, hipotireoidismo e hipogonadismo. ${ }^{10}$

O objetivo do presente estudo é relatar um caso de epifisiólise em uma paciente de 22 anos de idade com atraso de desenvolvimento sexual e com diagnóstico de hipogonadismo hipogonadotrófico congênito. A epifisiólise é uma 


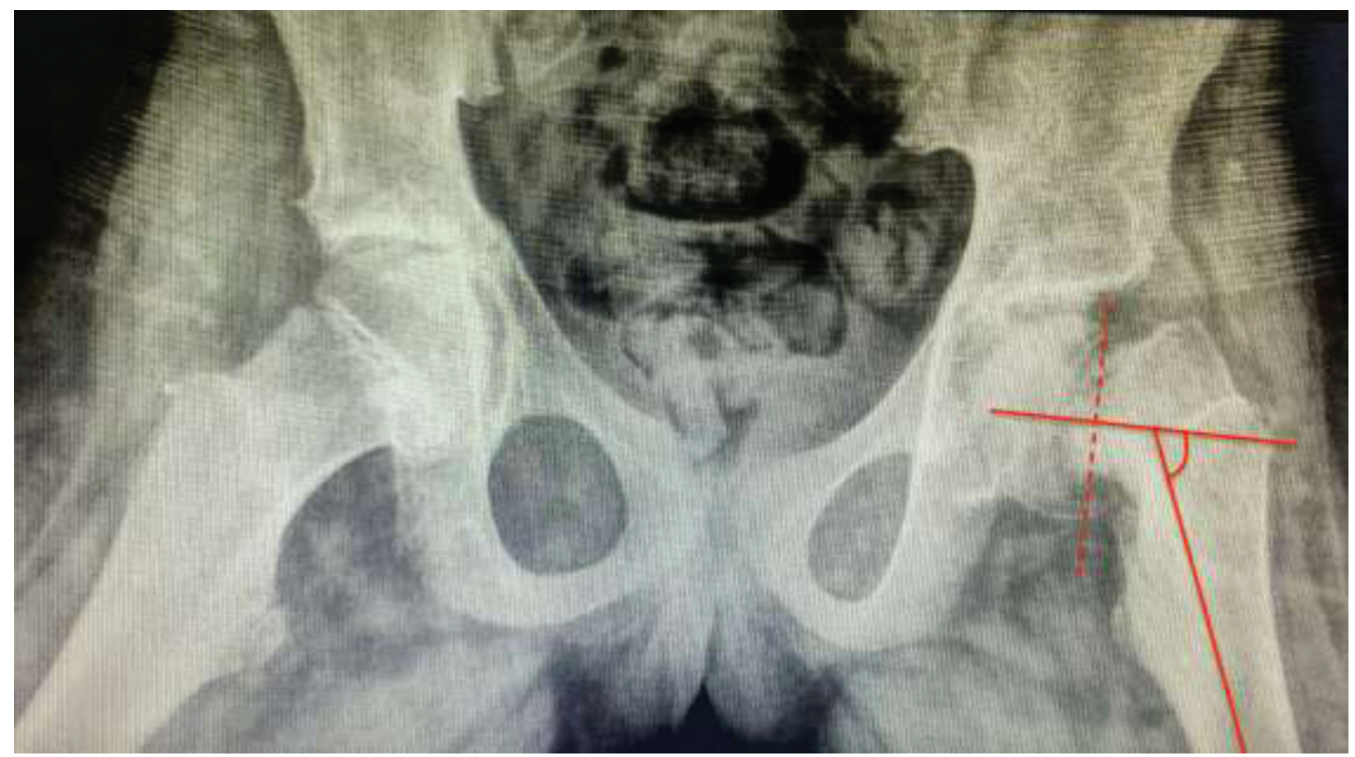

Fig. 2 Radiografia da pelve - incidência Lauenstein com mensuração do ângulo de Southwick do lado acometido.

doença rara que se caracteriza pela ausência de desenvolvimento puberal, decorrente de problemas na produção ou secreção do hormônio liberador de gonadotrofinas (GnRH). Laboratorialmente, são observadas baixas concentrações de esteroides sexuais (testosterona/estradiol) e valores reduzidos ou normais de gonadotrofinas hipofisárias (LH e FSH). Os demais hormônios hipofisários e a ressonância magnética hipotalâmica-hipofisária encontram-se normais. Quando associada à anosmia, tem-se a síndrome de Kallmann.

\section{Relato do Caso}

Uma paciente do sexo feminino, com 22 anos de idade, procurou atendimento ortopédico queixando-se de dor insidiosa no quadril há 3 meses, negando trauma. Referiu ter procurado atendimento duas vezes anteriormente e ter sido tratada com medicação, sem exames. A paciente negava dor no outro quadril.

De antecedente pessoal, ela relatou que não teve menarca e que fazia acompanhamento ambulatorial com ginecolo- gista por apresentar "útero infantil”. Caracteres secundários sexuais não desenvolvidos.

Apresentava o membro inferior esquerdo rodado externamente e dor à mobilização passiva do quadril com manobra de Drehmann positiva.

As radiografias da pelve ( - Figuras $\mathbf{1}$ e $\mathbf{2}$ ) evidenciaram as fises femorais proximais ainda abertas e o escorregamento do lado esquerdo.

Optamos pelo tratamento cirúrgico com fixação percutânea com parafuso canulado (-Figura 3). Pelo risco de ocorrência contralateral, foi sugerida a fixação, entretanto a paciente recusou.

Durante o acompanhamento, a paciente apresentou os exames prévios, com diagnóstico de hipogonadismo hipogonadotrófico congênito. A ultrassonografia pélvica apresentou útero com dimensões muito reduzidas $2,3 \times 0,9$ $\times 1,5 \mathrm{~cm}$ (volume de $2 \mathrm{~cm}^{2}$ ) - dimensões infantis. Os exames laboratoriais mostraram prolactina, TSH, T4 livre e GH dentro dos valores de normalidade. Testosterona total inferior a $10 \mathrm{ng} / \mathrm{dL}, \quad \mathrm{LH}<0,07 \mathrm{mUI} / \mathrm{mL}, \quad \mathrm{FSH}<0,30 \mathrm{mUI} / \mathrm{mL}$ e

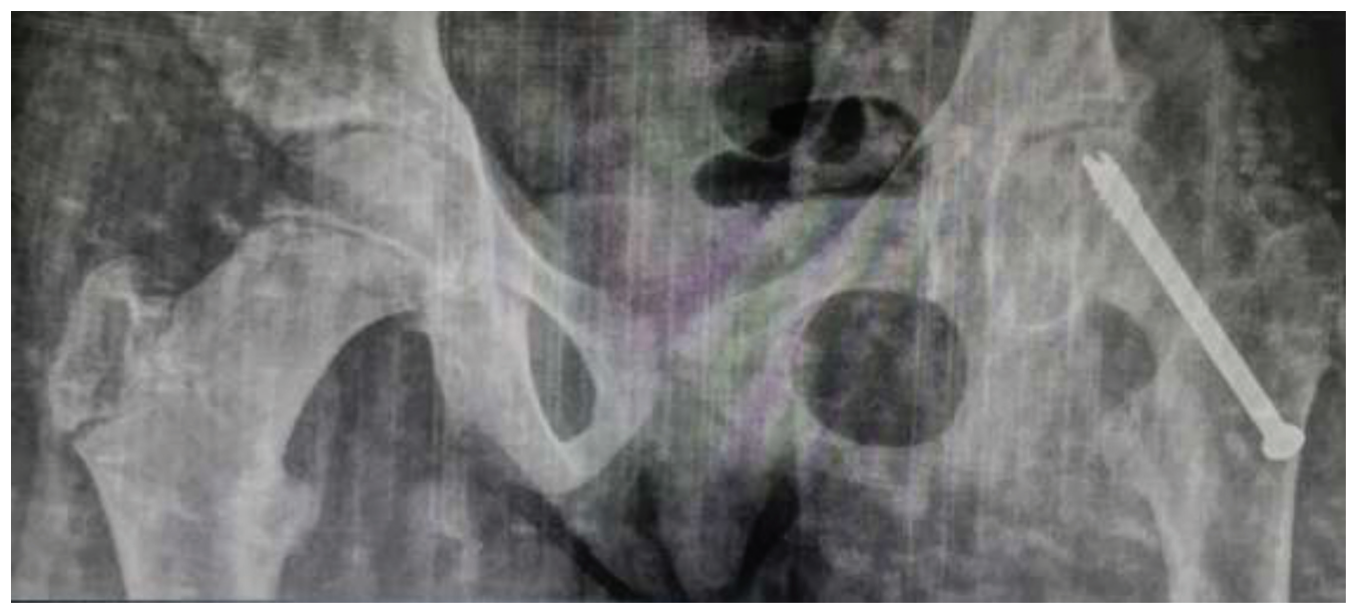

Fig. 3 Radiografia - incidência frontal, pós-operatória com fixação com parafuso canulado. 
estradiol $<11,80 \mathrm{pg} / \mathrm{mL}$ - todos valores bem abaixo da normalidade.

Após 3 meses, a paciente apresentou fechamento fisário do lado operado, e até 6 meses após a cirurgia não apresentou queixas de dor no lado contralateral.

\section{Discussão}

A epifisiólise é extremamente rara em adultos, e quando ocorre está associada a alguma doença que retarde o desenvolvimento sexual e fechamento fisário.

Por se tratar de um diagnóstico raro e de exclusão, muitas vezes é negligenciado e seu tratamento retardado, fazendo com que a real prevalência na população adulta seja desconhecida/subestimada. Acredita-se que muitos casos de osteoartrose do quadril em pacientes jovens sejam sequelas de casos não diagnosticados. ${ }^{5}$

Foi realizada uma revisão da literatura com os termos: epifisiólise, slipped capital femoral epiphysis, delayed slipped capital femoral epiphysis, e slipped capital femoral epiphysis in adults. Na literatura mundial existem 63 relatos de casos na população adulta e nenhum na literatura brasileira.

As doenças mais comumente associadas à ocorrência de epifisiólise na população adulta descritas nos relatos de caso são doenças hipofisárias/craniofaringioma, hipotireoidismo, e hipogonadismo, indo de encontro com outras revisões prévias.

Foram realizadas duas revisões anteriores da literatura. Macía-Villa et al. ${ }^{5}$ relataram um caso em uma paciente do sexo feminino de 47 anos de idade com uso crônico de corticosteroide, e realizaram uma revisão da literatura encontrando 60 casos; 17 com distúrbios hipofisários, 7 com causas endocrinológicas não-especificadas, 5 com hipotireoidismo e 4 casos de hipogonadismo.

Speirs et al. ${ }^{10}$ também fizeram um levantamento da literatura e encontraram associações semelhantes à revisão anterior.

Este relato de caso trata-se de uma doença rara na população adulta associada a hipogonadismo hipogonadotrófico congênito.

É importante considerar a possibilidade do diagnóstico em adultos com dores no quadril, principalmente dores crônicas, com história de doenças endocrinológicas ou alteração dos caracteres sexuais secundários.

\section{Contribuição dos autores}

A. P. N. foi responsável pela concepção e desenho, redação do artigo e aprovação final da versão submetida.

C. T. K. foi responsável pela concepção e desenho, redação do artigo e aprovação final da versão submetida.

\section{Conflito de Interesses}

Os autores declaram não haver conflito de interesses.

\section{Referências}

1 Mathew SE, Larson AN. Natural History of Slipped Capital Femoral Epiphysis. J Pediatr Orthop 2019;39(6, Supplement 1 Suppl 1): S23-S27

2 Otani T, Kawaguchi Y, Marumo K. Diagnosis and treatment of slipped capital femoral epiphysis: Recent trends to note. J Orthop Sci 2018;23(02):220-228

3 Huang YF, Wang LS, Zhang S, Gao YH, Liu JG, Qi X. Slipped capital femoral epiphysis in an adult with congenital hypopituitarism: A case report. Medicine (Baltimore) 2019;98(03): e13997

4 Georgiadis AG, Zaltz I. Slipped capital femoral epiphysis: how to evaluate with a review and update of treatment. Pediatr Clin North Am 2014;61(06):1119-1135

5 Macía-Villa CC, Sanchez-Lite I, Medina-Luezas J. Slipped capital femoral epiphysis in adults: case report and review of literature. Reumatismo 2016;68(01):40-47

6 Naseem H, Chatterji S, Tsang K, Hakimi M, Chytas A, Alshryda S. Treatment of stable slipped capital femoral epiphysis: systematic review and

7 Herngren B, Stenmarker M, Vavruch L, Hagglund G. Slipped capital femoral epiphysis: a population-based study. BMC Musculoskelet Disord 2017;18(01):304

8 Schur MD, Andras LM, Broom AM, et al. Continuing Delay in the Diagnosis of Slipped Capital Femoral Epiphysis. J Pediatr 2016; 177:250-254

9 Perry DC, Metcalfe D, Costa ML, Van Staa T. A nationwide cohort study of slipped capital femoral epiphysis. Arch Dis Child 2017; 102(12):1132-1136

10 Speirs JN, Morris SC, Morrison MJ III. Slipped Capital Femoral Epiphysis in an Adult Patient With Kabuki Syndrome. J Am Acad Orthop Surg Glob Res Rev 2019;3(10):e19.00084 